\title{
Amlexanox Inhibits Cerebral Ischemia-Induced Delayed Astrocytic High-Mobility Group Box 1 Release and Subsequent Brain Damage ${ }^{\mathbb{\Phi}}$
}

\author{
Sebok Kumar Halder and Hiroshi Ueda
}

\author{
Department of Pharmacology and Therapeutic Innovation, Nagasaki University Graduate School of Biomedical Sciences, \\ Nagasaki, Japan
}

Received September 25, 2017; accepted January 10, 2018

\begin{abstract}
High-mobility group box 1 (HMGB1) is increased in the cerebrospinal fluid (CSF) and serum during the early and late phases of brain ischemia and is known to contribute to brain damage. However, detailed characterization underlying cell type-specific HMGB1 release and pathophysiological roles of extracellularly released HMGB1 in ischemic brain remain unclear. Here, we examined cell type-specific HMGB1 release and the therapeutic potential of amlexanox, an inhibitor of nonclassical release, and of an anti-HMGB1 antibody against ischemic brain damage. HMGB1 depletion from neuronal nuclei was observed within 3 hours after transient middle cerebral artery occlusion (tMCAO), whereas the intracerebroventricular (i.c.v.) pretreatment with amlexanox blocked HMGB1 release from neurons, resulting in HMGB1 redistribution in the nuclei and cytoplasm. HMGB1 was selectively
\end{abstract}

released from astrocytes 27 hours after tMCAO and this HMGB1 release was blocked by late treatment with amlexanox (i.c.v.) 24 hours after tMCAO. Proximity extension assay revealed that the HMGB1 level was elevated in the CSF at 3 and 27 hours after tMCAO. This late treatment with amlexanox significantly protected the brain from ischemic damage, but its pretreatment 30 minutes before $\mathrm{TMCAO}$ failed to show any protection. The late treatment (i.c.v.) with anti-HMGB1 antibody 24 hours after tMCAO also ameliorated ischemic brain damage 48 hours after tMCAO. Thus, the inhibition of brain damage by late treatment with amlexanox or anti-HMGB1 antibody indicates that late HMGB1 release plays a role in the maintenance of stroke-induced brain damage, and the inhibition of this release would be a novel therapeutic target for protection of ischemic brain damage.

\section{Introduction}

High-mobility group box 1 (HMGB1), a nuclear protein, is ubiquitously expressed throughout mammalian tissues including the brain. It is involved in diverse intracellular functions, such as nucleosome sliding, recombination, and regulation of transcription (Lange et al., 2008; Stros, 2010; Harris et al., 2012). In addition to its intracellular roles, extracellular functions of HMGB1 in cell proliferation, migration, differentiation, tissue regeneration, and pathogenesis of inflammation and diseases have also been reported (Erlandsson Harris and Andersson, 2004; Palumbo et al., 2009; Andersson and Tracey, 2011; Tang et al., 2011). Previous studies showed that HMGB1 is released either actively from monocytes and macrophages or passively from injured/damaged cells following ischemic/necrotic stress (Lotze and Tracey, 2005; Tang et al., 2011), and this extracellularly

This study was supported by Grants-in-Aid for Scientific Research (to H.U.) from the Ministry of Education, Culture, Sports, Science and Technology (MEXT) and Health and Labor Sciences Research Grants (to H.U.) on Research from the Ministry of Health, Labor and Welfare. Authors of this manuscript have no conflict interest to report.

http://.doi.org/10.1124/jpet.117.245340.

S This article has supplemental material available at jpet.aspetjournals.org. released HMGB1 functions as a cytotoxic mediator through activation of receptor for advanced glycation end products (AGEs) and Toll-like receptors (TLR)-2 and -4 (Yu et al., 2006; Klune et al., 2008; Muhammad et al., 2008). Thus, HMGB1 is known as a representative member of damage-associated molecular patterns (DAMPs) family molecules.

In the brain, HMGB1 is rapidly released from neurons or astrocytes by various stresses, including ischemia and immunochemical stress, whereas ischemia-induced HMGB1 release from microglia occurs as late as 1 week after the stress (Kim et al., 2006; Hayakawa et al., 2008). Thus, released HMGB1 is known to cause a neurodegeneration, and the neutralization of extracellular HMGB1 prevents the progression of ischemic brain damage (Liu et al., 2007; Yang et al., 2010). For an improved understanding of strategic treatments using small molecules against ischemic brain damage, it is important to examine which cell types and what time points are responsible for the HMGB1 release in terms of brain damage.

One strategy to suppress the inflammatory actions of DAMPs could be the use of an inhibitor of DAMPs release, though the manner of DAMPs release is considered to be performed in an unidentified nonclassical release. Previous studies reported that amlexanox (2-amino-7-isopropyl-5-oxo-5H-[1]benzopyrano[2,3-b]

ABBREVIATIONS: ANOVA, One-way analysis of variance; CSF, cerebrospinal fluid; DAB, 3,3'-diaminobenzidine tetrahydrochloride; DAMP, damage-associated molecular pattern; GFAP, glial fibrillary acidic protein; HMGB1, high-mobility group box 1; i.c.v., intracerebroventricular; NeuN, neuronal nuclei; PBS, phosphate-buffered saline; PEA, proximity extension assay; PFA, paraformaldehyde; ProT $\alpha$, prothymosin alpha; tMCAO, transient middle cerebral artery occlusion; TTC, 2,3,5-triphenyltetrazolium chloride. 
pyridine-3-carboxylic acid), an antiallergy drug, inhibits the stress-induced nonclassical release of representative DAMPs molecule interleukin-1 $\alpha$ (IL-1 $\alpha$ ) (Okada et al., 2003; Mohan and $\mathrm{Yu}, 2011)$ and neurotrophic or neuroprotective DAMPs molecules, such as fibroblast growth factor-1 (FGF-1) (Mouta Carreira et al., 1998; Landriscina et al., 2000) and prothymosin alpha $(\operatorname{ProT} \alpha)$ (Fujita and Ueda, 2007; Fujita et al., 2009; Matsunaga and Ueda, 2010; Halder et al., 2012, 2015; Ueda et al., 2012). In the present study, we examined ischemia-induced HMGB1 release from the temporo-spatial point of view, in an attempt to evaluate the effects of amlexanox on HMGB1 release and brain damage.

\section{Materials and Methods}

Animals. Male C57BL/6J mice (6-weeks-old, 20-24 g body weight) were purchased from Tagawa Experimental Animals (Nagasaki, Japan). Mice were housed in a room maintained at a temperature of $21 \pm 2{ }^{\circ} \mathrm{C}$ and relative humidity of $55 \pm 5 \%$ with a 12-hour light/dark cycle. Mice had free access to both standard laboratory diet and tap water. All procedures were approved (approval number: 1104190914) by Nagasaki University Animal Care Committee (Nagasaki, Japan).

Amlexanox and Anti-HMGB1 Antibody Treatments. Amlexanox was purchased from Takeda Pharmaceutical Company Ltd. (Osaka, Japan) and dissolved in autoclaved phosphate-buffered saline (PBS). Anti-HMGB1 antibody (rabbit polyclonal) was purchased from Abcam (Cambridge, MA) and diluted in autoclaved PBS. Intracerebroventricular (i.c.v.) injection takes advantage of the animal ventricular system that bathes the whole brain, allowing widespread distribution of the injected molecules. In i.c.v. injection, a small dose of injected molecules is sufficient to reach the molecular target within brain tissue. In contrast, systemically administered molecules may fail to reach therapeutic levels in the brain at different time points after cerebral ischemia, owing to their degradation in blood. Considering these issues, we performed i.c.v. injection using a Hamilton syringe to get a direct effect of amlexanox or anti-HMGB1 antibody on the brain tissue as well as to minimize any other peripheral targets. The following mice groups were used throughout the study: Mice received amlexanox $(10 \mu \mathrm{g} / 5 \mu$ l, i.c.v. 30 minutes before 1 -hour transient middle cerebral artery occlusion (tMCAO) $(n=6)$ or 15-minute tMCAO $(n=6)$. Mice received amlexanox $(10 \mu \mathrm{g} / 5 \mu \mathrm{l}$, i.c. v.) 24 hours after 1 -hour tMCAO $(n=6)$ or 30 -minute tMCAO $(n=9)$. Mice received amlexanox $(10 \mu \mathrm{g} / 5 \mu \mathrm{l}$, i.c.v. $) 48$ hours after 30 -minute $\operatorname{tMCAO}(n=4)$. Amlexanox was also injected $(10 \mu \mathrm{g} / 5 \mu$ l, i.c.v. $)$ in nonischemic mice $(n=6)$. Mice received anti-HMGB1 antibody ( 1 or $3 \mu \mathrm{g} / 5 \mu \mathrm{l}$, i.c.v.) 24 hours after 30 -minute tMCAO $(n=3)$ or 1 -hour tMCAO $(n=6)$. Ischemic (tMCAO) vehicles were prepared by PBS injection (i.c.v.). Mice receiving neither surgery (tMCAO) nor injection were referred to as the untreated group, which was used as control to compare with ischemic vehicle.

Middle Cerebral Artery Occlusion Model. tMCAO was performed according to a previously described standard protocol (Egashira et al., 2004). Briefly, mice were first anesthetized with $2 \%$ isoflurane (Mylan, Tokyo, Japan) and during the period of occlusion maintained at $1 \%$ isoflurane with a face mask. Rectal temperature was monitored and maintained at $37^{\circ} \mathrm{C}$ by a thermostatically controlled heating blanket throughout the surgical procedure. After a midline incision of neck, the middle cerebral artery was transiently occluded using 8-0 monofilament nylon surgical suture (Natsume Co. Ltd., Tokyo, Japan) coated with silicon (Xantopren; Bayer Dental, Osaka, Japan) that was inserted through the left common carotid artery and advanced into the left internal carotid artery. Anesthesia was immediately excluded after occlusion and the occluded mice were kept in a cage on a warm plate at $37^{\circ} \mathrm{C}$. After 1 hour of occlusion (tMCAO), mice were briefly reanesthetized with $1 \%$ isoflurane using a face mask and the monofilament was withdrawn for reperfusion. After removal of anesthesia, mice were further kept on a warm plate at $37^{\circ} \mathrm{C}$ for 1-2 hours and thereafter returned to the home cage. As the siliconcoated nylon suture also plugs the branch from the middle cerebral artery that supplies blood to the hippocampus and because of small brain size, ischemia-induced brain damage was also observed in the hippocampus. Likewise, 15- or 30-minute tMCAO were prepared by occlusion of the middle cerebral artery for 15 or 30 minutes, respectively. Cerebral blood flow was monitored by insertion of the probe (diameter 0.5 mm, ALF2100; Advance Co., Tokyo, Japan) of a Doppler flowmeter (ALF21; Advance Co.) into the left striatum through a guide cannula.

Collection of Cerebrospinal Fluid. Cerebrospinal fluid (CSF) was collected from the cisterna magna in mouse brain according to a previously described protocol (Liu and Duff, 2008). The glass capillary tube with an inner tip diameter of $0.3-0.5 \mathrm{~mm}$ was manually prepared by heating the tip of the Pasteur pipette (Soda-lime glass, IK-PASS5P; Asahi Glass Co., Ltd, Tokyo, Japan) on a low flame of the gas burner. Mice were deeply anesthetized with intraperitoneal injection of sodium pentobarbital ( $50 \mathrm{mg} / \mathrm{kg}$ ), and then placed on a heating plate to maintain body temperature at $37^{\circ} \mathrm{C}$. Under dissection microscope, a sagittal incision of the skin was made inferior to the occiput and the subcutaneous tissue and muscles (biventer cervicis and rectus capitis dorsalis major) were separated by blunt dissection. Mice were laid down so that the head formed a nearly $135^{\circ}$ angle with the body. The dura mater of the cisterna magna was exposed as a glistening and clear reverse triangle through which the medulla oblongata with a major blood vessel (arteria dorsalis spinalis) and CSF space were visible. The tip of the glass capillary tube was inserted into the cisterna magna through the dura mater, and CSF was drawn into the capillary tube. CSF (approximately $3 \mu \mathrm{l} /$ mouse brain) was immediately transferred from the capillary tube into the $1.5-\mathrm{ml}$ sample Eppendorf tube on dry ice and then stored at $-80^{\circ} \mathrm{C}$.

Proximity Extension Assay. Proximity extension assay (PEA) (Proseek; Olink Bioscience, Uppsala, Sweden) was performed to measure the HMGB1 level in the CSF, according to the manufacturer's supplied kits and standard guidelines (http://www.olink.com) as described previously (Hjelm et al., 2011). To generate Proseek probes, polyclonal anti-HMGB1 antibody (Abcam) was divided into two fractions $(1 \mathrm{mg} / \mathrm{ml})$ and individually conjugated with two different DNA oligo sequences A and B to produce anti-HMGB1 antibody-oligo primer A and antibody-oligo primer B. The Proseek probes were then incubated with $1 \mu \mathrm{l}$ of CSF for 2 hours at $20^{\circ} \mathrm{C}$ and allowed to bind pairwise to the target HMGB1 in the CSF. The Proseek probes were also incubated with series of $70,100,300 \mathrm{pM}, 1$ and $3 \mathrm{nM}$ Strep-tagged mouse HMGB1 and a zero buffer to generate a calibration curve. When two Proseek probes come into close proximity, a new polymerase chain reaction (PCR) target sequence is generated by proximity-dependent DNA polymerization. After incubation of the probes, the HMGB1 level in the CSF was detected and quantified by standard real-time PCR. The cycle threshold $(\mathrm{Ct})$ values obtained by real-time PCR were then converted to concentration. The calibration curve generated from a dilution series of Strep-tagged HMGB1 was used to evaluate the sensitivity and quantification of HMGB1 concentration in the CSF.

Immunohistochemistry. Mice were deeply anesthetized with sodium pentobarbital $(50 \mathrm{mg} / \mathrm{kg}$, i.p.) and perfused transcardially with $0.1 \mathrm{M}$ PBS (pH 7.4) and 4\% paraformaldehyde (PFA). The brain was removed, post-fixed in $4 \%$ PFA, and transferred to $25 \%$ sucrose solution overnight before being frozen in cryoembedding compound for preparation of coronal brain sections at $30-\mu \mathrm{m}$ thickness.

Immunohistochemical analysis was performed following a previously reported protocol (Halder et al., 2012). To perform 3,3'diaminobenzidine tetrahydrochloride (DAB) immunostaining of HMGB1, the floating brain sections were incubated with $1 \% \mathrm{H}_{2} \mathrm{O}_{2}$ and washed with $0.1 \%$ Triton X-100 in PBS (PBST). Following incubation with blocking buffer containing $2 \%$ bovine serum albumin (BSA) in $0.1 \%$ PBST, the sections were incubated with anti-HMGB1 IgG (1:100, rabbit polyclonal; Abcam) overnight at $4^{\circ} \mathrm{C}$. The sections were then incubated with biotin-conjugated secondary antibody 
(1:300; Vector Laboratories, Burlingame, CA) and subsequently treated with avidin-biotin peroxidase solution (VECTASTAIN ABC kit; Vector Laboratories). The HMGB1 reactivity was visualized by incubation of the sections with a solution containing $0.02 \%$ DAB (Dojindo, Kumamoto, Japan), 0.005\% $\mathrm{H}_{2} \mathrm{O}_{2}$ (WAKO, Osaka, Japan) in $0.05 \mathrm{M}$ Tris-HCl buffer ( $\mathrm{pH} 7.6), 1 \%$ cobalt chloride $\left(\mathrm{CoCl}_{2}\right)$, and $1 \%$ nickel sulfate $\left(\mathrm{NiSO}_{4}\right)$ solution (Sigma-Aldrich, St. Louis, MO). The sections were dehydrated through a series of ethanol solutions, fixed in xylene, and cover-slipped with Fisher Scientific Permount (Thermo Fisher Scientific, Waltham, MA). Immunoreactivity of HMGB1positive cells (black reaction products) was analyzed using a BZ-8000 microscope with BZ image measurement software (KEYENCE, Osaka, Japan). For fluorescence immunostaining, the brain sections were washed with $\mathrm{K}^{+}$-free PBS and sequentially incubated with 50 and $100 \%$ methanol. The sections were treated with blocking buffer ( 2 or $3 \%$ BSA in $0.1 \%$ PBST) and incubated overnight at $4^{\circ} \mathrm{C}$ with following primary antibodies: anti-NeuN (1:100; mouse monoclonal IgG $_{1}$; Chemicon, Temecula, CA); anti-GFAP (1:400; mouse monoclonal $\mathrm{IgG}_{1}$;Chemicon); and rabbit polyclonal (1:500; Dako, Glostrup, Denmark), anti-Iba-1 (1:500; goat polyclonal IgG ; Abcam), and anti-HMGB1 IgG (1:100; rabbit polyclonal IgG; Abcam). The sections were then incubated with Alexa Fluor 594-conjugated antirabbit IgG, Alexa Fluor 488-conjugated anti-mouse IgG, Alexa Fluor 488-conjugated anti-goat IgG (1:300; Molecular Probes, Eugene, OR). The nuclei were visualized with Hoechst 33342 (1:10,000; Molecular Probes). The sections were washed with PBS and cover-slipped with Thermo Scientific PermaFluor (Thermo Fisher Scientific). Images were collected using a BZ-8000 microscope with BZ image measurement software (KEYENCE).

Cell Counting. Measurement of HMGB1-positive cells in the brain was carried out according to a previously described protocol (Halder et al., 2012). Briefly, the counting of HMGB1-positive cells in the coronal brain sections was performed in bright-field images using a BZ-8000 microscope with BZ image measurement software (KEYENCE). Cell-type-specific markers with clearly visible nuclei using Hoechst 33342 staining were used to detect HMGB1 signals in neuronal nuclei (NeuN)-positive neurons, ionized calcium-binding adapter molecule-1 (Iba1)-positive microglia, and glial fibrillary acidic protein (GFAP)-positive astrocytes. The number of HMGB1-positive neurons, astrocytes and microglia were stereologically counted (bregma 0.68-2.08) in the square fields (approximately $250 \times 250 \mu \mathrm{m}$ ) of untreated brain $(n=3)$, vehicle-treated tMCAO brain $(n=3)$, and amlexanox-treated tMCAO brain $(n=3)$ and were normalized to the untreated brain $(n=3)$. The quantification was expressed as the average percentage of the total number of cell-type-specific HMGB1positive cells in the three coronal brain sections per mouse.

Triphenyltetrazolium Chloride Staining. To perform 2,3,5triphenyltetrazolium chloride (TTC) staining, the brain was quickly removed and sectioned coronally to 1-mm thickness. TTC (SigmaAldrich) was dissolved in 0.9\% physiologic saline (Otsuka Pharmaceutical Co. Inc., Tokyo, Japan). Brain slices were washed with PBS and then incubated with 2\% TTC in the dark for 15-20 minutes at room temperature $\left(25^{\circ} \mathrm{C}\right)$. Brain slices were further incubated with $4 \%$ PFA overnight at $4^{\circ} \mathrm{C}$. Images of brain slices were obtained by scanner (EPSON GT-9700F) and infarct volume was measured by Image $\mathrm{J}$ software (NIH, Bethesda, MD).

Behavioral Assessments. According to a previously described protocol (Wang et al., 2015; Maeda et al., 2016), clinical (neurologic) scores were measured after tMCAO using a six-point (0-5) scale: $0=$ no observable deficits; 1 = failure to extend the forepaw fully; $2=$ circling to the left; $3=$ falling to the left side; $4=$ no spontaneous movement; $5=$ death. In this study, 0.5 points were added to each score when the motor dysfunction was severe for scores between 1 and 4 .

Statistical Analysis. Data are means \pm S.E.M. Two independent groups were compared using Student's $t$ test. Multiple groups were compared using Dunnett's multiple comparison tests after one-way analysis of variance (ANOVA). $P<0.05$ was considered significant.

\section{Results}

Ischemia-Induced Elevation of HMGB1 Level in the CSF. To confirm whether HMGB1 is released from brain after cerebral ischemia, CSF was collected from the cisterna magna at 3 and 27 hours after the 1-hour tMCAO. As shown in Fig. 1A, the detection limit of HMGB1 level in the PEA was below $2 \mathrm{pM}$. The PEA study revealed that the HMGB1 levels at 3 and 27 hours after tMCAO were significantly higher than in the untreated mice (Fig. 1B).

Amlexanox Reverses Ischemia-Induced Depletion of HMGB1 in the Brain. The HMGB1 immunoreactivities by $\mathrm{DAB}$ staining were widely distributed in nuclei in the brain cells of cerebral cortex, striatum, and hippocampus (Fig. 2, A-C). The tMCAO treatment of 1 hour depleted the HMGB1
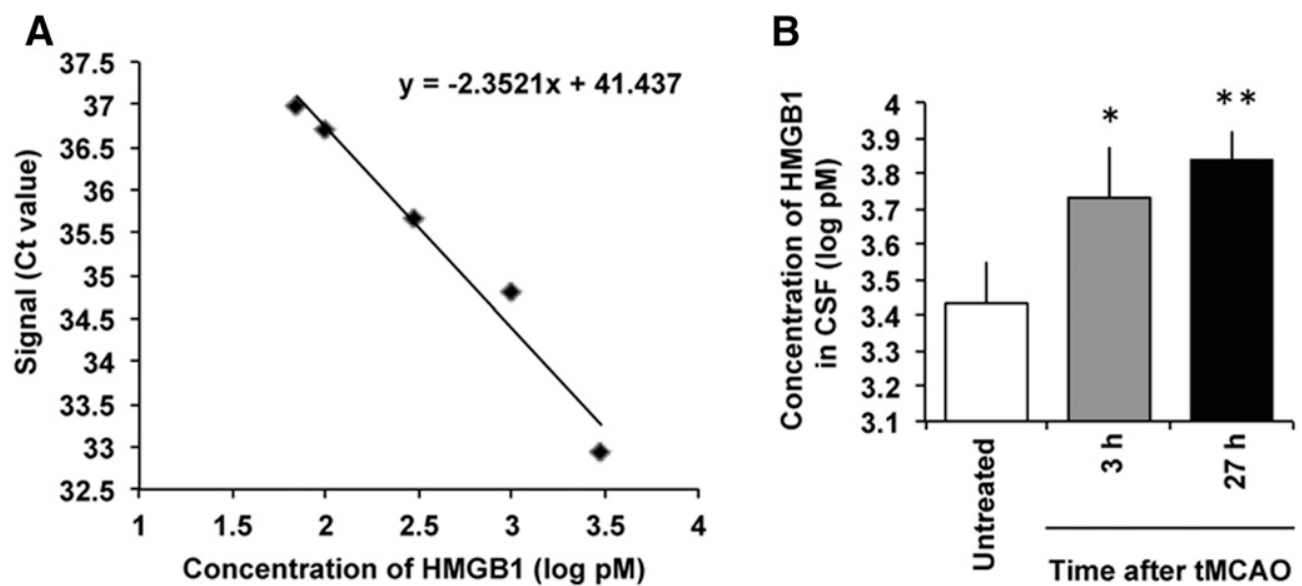

Fig. 1. HMGB1 is elevated in the cerebrospinal fluid following cerebral ischemia. (A) Calibration curve for HMGB1. Proximity extension assay (PEA) was performed to generate the calibration curve using 70,100, and $300 \mathrm{pM}$, and 1 and $3 \mathrm{nM}$ Strep-tagged mouse HMGB1 and a zero buffer. Average cycle threshold (Ct) values are plotted on the $y$-axis and concentrations of HMGB1 on the $x$-axis. (B) Cerebrospinal fluid (CSF) was withdrawn from the cisterna magna of mouse brain at 0 (untreated), 3, and 27 hours after 1-hour tMCAO, and HMGB1 level was measured using PEA. HMGB1 level in the $\mathrm{CSF}$ was measured by converting the $\mathrm{Ct}$ values into the concentrations. Data are means \pm S.E.M. $* P<0.05$ vs. untreated mice, $* * P<0.01$ vs. untreated mice; $n=3$ in each group. 
immunoreactivities from most cells in these three regions in the brain at 3 hours after tMCAO, though some cells still retained an HMGB1 signal. Although the cell type showing HMGB1 release 3 hours after tMCAO was not determinable by this method, it appears it was astrocytes in the image in Fig. 2C, which shows a complete depletion of HMGB1 in the hippocampal pyramidal neuronal layer of cornu ammonis (CA1). The decreased HMGB1 levels were largely recovered at 27 hours after tMCAO (Fig. 2, A-C). The pretreatment with amlexanox $(10 \mu \mathrm{g} / 5 \mu$ l, i.c.v.) 30 minutes prior to tMCAO reversed the decrease in neuronal nuclear HMGB1 levels at 3 hours, though there was no change in the HMGB1 levels at 27 hours (Fig. 2, A-C).

Amlexanox-Reversible Blockade of Rapid HMGB1 Depletion in Neurons. When the section of cortex was costained with anti-NeuN and anti-HMGB1 antibodies, all neurons were found to contain HMGB1 within their nuclei, as shown in Fig. 3A. The HMGB1 immunoreactivities were completely lost from neurons at 3 hours after 1-hour tMCAO (Fig. 3A). The pretreatment with amlexanox ( $10 \mu \mathrm{g} / 5 \mu$ l, i.c.v.) 30 minutes prior to tMCAO also reversed the decrease of HMGB1 levels at 3 hours, but HMGB1 immunoreactivities were found both in the cytosol and nucleus. As seen in Fig. 3A, most of the HMGB1 immunoreactivity was recovered only in the nuclei at 27 hours after tMCAO. The pretreatment with amlexanox did not show any change in the HMGB1 level at 27 hours, but HMGB1 signals were found only in the nucleus. Quantitative analysis evaluating the number of HMGB1positive neurons also supported the significant amlexanoxreversible HMGB1 release from cortical neurons (Fig. 3B). Similar amlexanox-reversible HMGB1 release from striatal and hippocampal neurons was also observed at 3 hours after MCAO (Fig. 3, C-E).

Late Release of Astroglial HMGB1 Is Blocked by Amlexanox. As shown in the untreated preparation (Fig. 4,
A and B), less intensive HMGB1-positive cells appear to be GFAP-positive astrocytes. The tMCAO stress for 1 hour caused a depletion of HMGB1 signals from GFAP-negative cells at 3 hours after tMCAO but not from GFAP-positive astrocytes, where HMGB1 was retained in the nucleus (Fig. 4, $\mathrm{B}$ and $\mathrm{C})$. The nuclear HMGB1 signal in astrocytes was still observed at 18 hours after tMCAO but was completely lost at 27 hours after tMCAO (Fig. 4C). When amlexanox ( $10 \mu \mathrm{g} / 5 \mu \mathrm{l}$, i.c.v.) was pretreated 30 minutes before or 24 hours after tMCAO, intense HMGB1 signals still remained in the nuclei in astrocytes at 3 or 27 hours, respectively (Fig. 4, B and C). The quantitatively significant decrease in the number of HMGB1-positive astrocytes and its recovery by amlexanox are shown in Fig. 4C. As seen in Fig. 4D, HMGB1 immunoreactivities were recovered only in the nuclei of astrocytes at 48 and 96 hours after tMCAO. The amlexanox treatment 24 hours after tMCAO did not show any change in the HMGB1 levels at 48 and 96 hours, but all HMGB1 signals were observed only in the nuclei of astrocytes (Fig. 4D). The tMCAO-induced HMGB1 release from the nuclei of astrocytes as late as 27 hours after the tMCAO, and its reversal by amlexanox, was also observed in preparations of striatum and stratum radiatum (str rad), as shown in Supplemental Fig. 1, A-D.

Ischemia-Induced Persistent Expression of HMGB1 in Microglia. As shown in untreated preparations of Fig. 5, $\mathrm{A}$ and $\mathrm{B}$, Iba-1-positive microglia residing in the somatosensory cortex of untreated mice have no HMGB1immunoreactivities. However, cerebral ischemia (1-hour tMCAO) caused an expression of HMGB1 in nuclei of cortical microglia on the ipsilateral side at 3 hours after tMCAO, and the expression became more intense at 27 hours after tMCAO (Fig. 5B). The pretreatment with amlexanox 30 minutes before or 24 hours after tMCAO had no effect on tMCAOinduced HMGB1 expression in the nuclei of microglia at 3 or

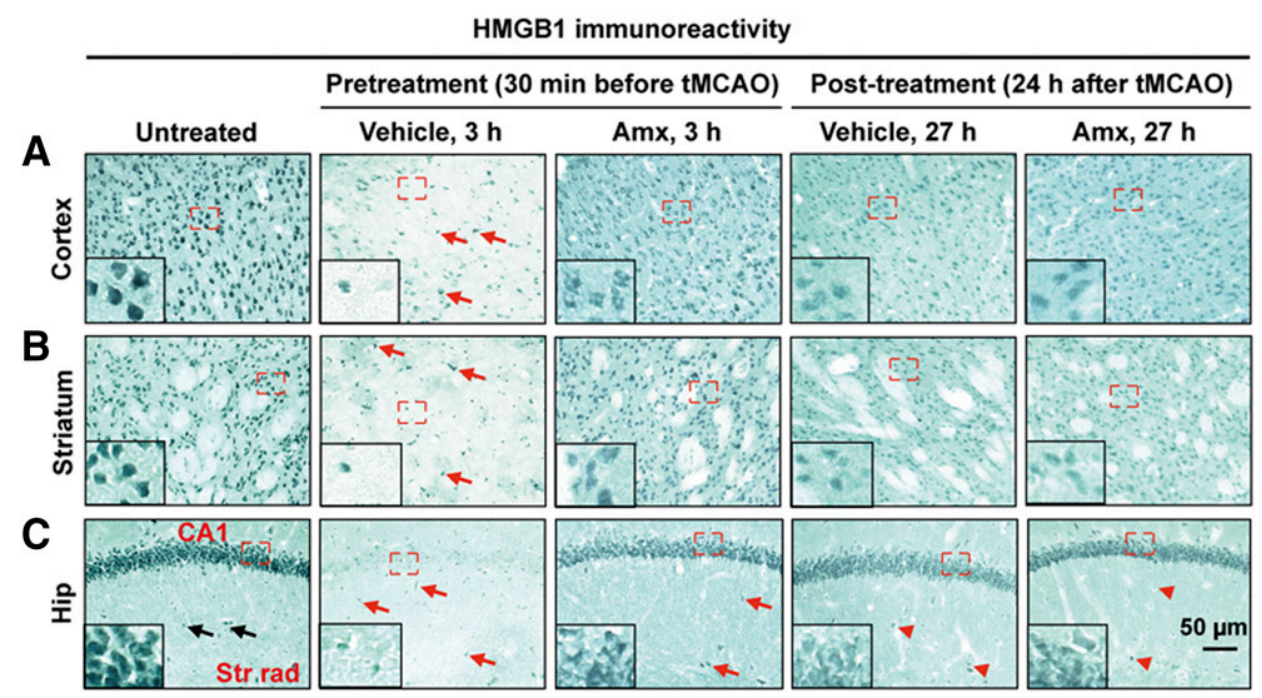

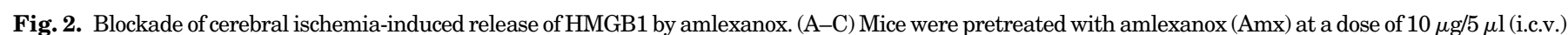

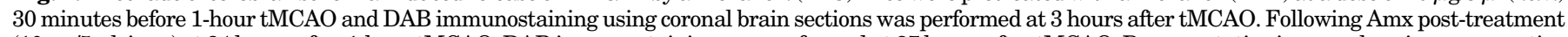

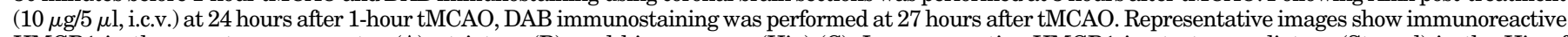

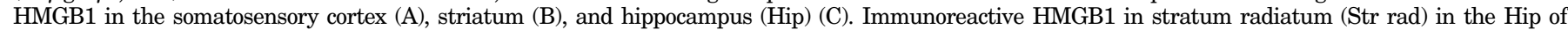

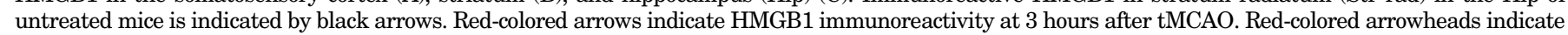

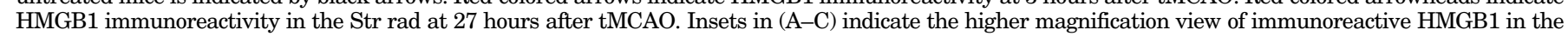
cortex (A), striatum (B) and cornu ammonis (CA1) of Hip (C) noted by red squares. $n=3$ in each group. 
$\underline{A}$

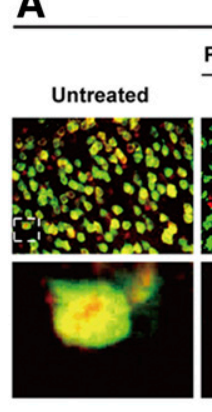

C__ Neun \& HMGB1

Pretreatment ( $30 \mathrm{~min}$ before tMCAO)

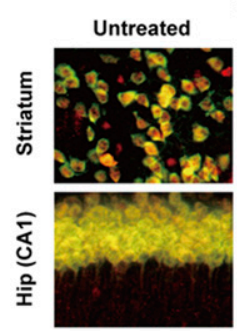

Cortex, NeuN \& HMGB1
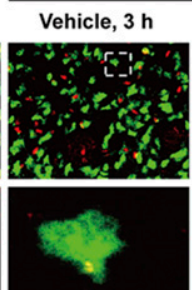

Vehicle, $3 \mathrm{~h}$
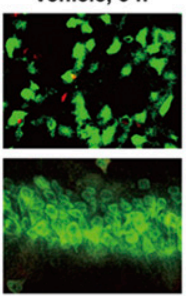

Pretreatment ( $30 \mathrm{~min}$ before tMCAO) Post-treatment ( $24 \mathrm{~h}$ after $\mathrm{tMCAO}$ )
Amx, $3 \mathrm{~h}$

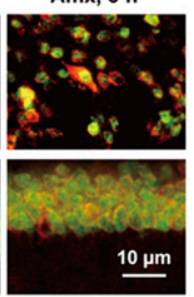

$\# \#$

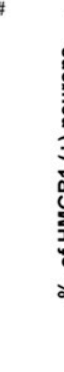

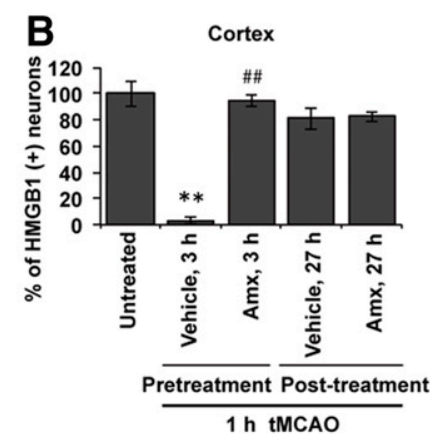

$1 \mathrm{~h}$ tMCAO

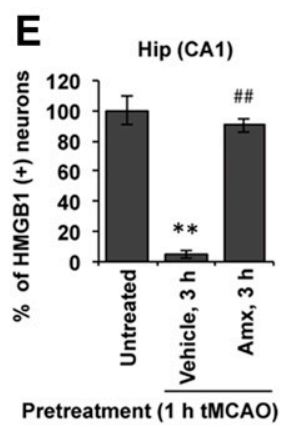

Fig. 3. Amlexanox-reversible blockade of HMGB1 release from neurons following ischemia. (A). Mice were pretreated with amlexanox (Amx) at a dose of $10 \mu \mathrm{g} / 5 \mu \mathrm{l}$ (i.c.v.) 30 minutes before 1-hour tMCAO, and immunostaining of NeuN and HMGB1 (NeuN, green; HMGB1, red; merge, yellow) in the somatosensory cortex using coronal brain sections was performed at 3 hours after tMCAO. Following Amx post-treatment (10 $\mu$ g/5 $\mu$ l, i.c.v. $)$ at 24 hours after 1-hour tMCAO, immunostaining of NeuN and HMGB1 was performed at 27 hours after tMCAO. The higher magnification views in the lower panels show HMGB1 localization in neurons noted by dotted squares in the upper panels. (B) Quantitative analysis of the number of HMGB1-positive neurons in the somatosensory cortex. Data are means \pm S.E.M. One-way ANOVA, Dunnett's multiple comparison tests, ${ }^{* *} P<0.01$ vs. untreated mice, ${ }^{\# \#} P<0.01$ vs. ischemic vehicle (3 hours). (C) Following Amx pretreatment (10 $\mu \mathrm{g} / 5 \mu$ l, i.c.v.) 30 minutes before 1-hour tMCAO, immunostaining of NeuN and HMGB1 (NeuN, green; HMGB1, red; merge, yellow) in the striatum (upper panels) and CA1 of hippocampus (lower panels) was performed 3 hours after tMCAO. (D) Quantitative analysis of HMGB1positive neurons in the striatum. Data are means \pm S.E.M. One-way ANOVA, Dunnett's multiple comparison tests, ${ }^{* *} P<0.01$ vs. untreated mice, ${ }^{\# \#} P<0.01$ vs. ischemic vehicle (3 hours). (E) Quantitative analysis of HMGB1-positive neurons in CA1 in the hippocampus (Hip). Data are means \pm S.E.M. One-way ANOVA, Dunnett's multiple comparison tests, ${ }^{* *} P<0.01$ vs. untreated mice, ${ }^{\# \#} P<0.01$ vs. ischemic vehicle (3 hours); $n=3$ in each group.

27 hours, respectively (Fig. 5B). Quantitative analysis revealed that tMCAO increased the number of HMGB1-positive microglia, but amlexanox did not affect the population at 3 and 27 hours (Fig. 5C). Similar negative effects of amlexanox on microglial HMGB1 expression at 48 and 96 hours were observed after the amlexanox treatment at 24 hours after tMCAO (Fig. 5D).

Pretreatment with Amlexanox Exacerbates the Ischemic Brain Damage. Following amlexanox pretreatment (10 $\mu \mathrm{g} / 5 \mu \mathrm{l}$, i.c.v.) 30 minutes before lethal cerebral ischemia (1-hour tMCAO), maximum infarct volume was obtained at 24 hours after tMCAO in either vehicle-pretreated or amlexanoxpretreated mice, as shown by TTC staining (Fig. 6, A and B). As it is difficult to evaluate the effect of amlexanox against ischemiainduced brain damage using the 1-hour tMCAO model, we used a mild cerebral ischemia model (15-minute tMCAO) to evaluate the beneficial effects of amlexanox. TTC staining revealed that infarct volume was significantly increased at day 1 (24 hours) after 15-minute tMCAO in amlexanox-pretreated (30 minutes before tMCAO) mice, compared with ischemic vehicle (Fig. 6, C and D). Clinical score was also significantly increased at day 1 (24 hours) after 15-minute tMCAO in amlexanox-pretreated mice, compared with ischemic vehicle (Fig. 6E). However, any changes in behaviors or in the infarct volume were not observed following treatment with amlexanox in nonischemic mice (Fig. 6E).

Late Treatment with Amlexanox Ameliorates the Ischemic Brain Damage. As mice show poor survival 4-6 days after 1-hour tMCAO (Halder et al., 2013), we used a 30-minute tMCAO model for behavioral assessments every day for 7 days to evaluate the beneficial effects of amlexanox late treatment. When mice were treated with amlexanox at 24 hours after 30-minute tMCAO, infarct volume was significantly decreased at 48 hours after tMCAO (Fig. 7, A and B). Clinical score was also significantly decreased at day 4 (96 hours) after 30-minute tMCAO in amlexanox post-treated (24 hours after tMCAO) mice, but the clinical score was gradually increased from day 5 and continued to day 7 after tMCAO (Fig. 7C). There was no change in infarct volume and clinical score at 72 hours after 30-minute tMCAO when mice were treated with amlexanox at 48 hours after tMCAO (Fig. 7, D-F).

Late Treatment with Anti-HMGB1 Antibody Reduces the Ischemic Brain Damage. To investigate whether ischemia-induced brain damage is inhibited by neutralization of released HMGB1, mice were treated with polyclonal antiHMGB1 antibody ( 1 or $3 \mu \mathrm{g} / 5 \mu \mathrm{l}$, i.c.v.) at 24 hours after 30-minute tMCAO or 1-hour tMCAO. TTC staining revealed that infarct volume was markedly increased 48 hours after mild cerebral ischemia (30-minute tMCAO), whereas posttreatment ( 24 hours) with $3 \mu \mathrm{g}$ of anti-HMGB1 antibody (but not $1 \mu \mathrm{g}$ ) significantly reduced the infarct volume at 48 hours after 30-minute tMCAO (Fig. 8, A and B). Post-treatment with anti-HMGB1 antibody ( $3 \mu \mathrm{g} / 5 \mu$ l, i.c.v. $)$ at 24 hours after lethal cerebral ischemia (1-hour tMCAO) also significantly reduced infarct volume 48 hours after tMCAO, compared with vehicletreated ischemic mice (Fig. 8, C and D). Moreover, the clinical score was significantly decreased through 7 days after 1-hour 


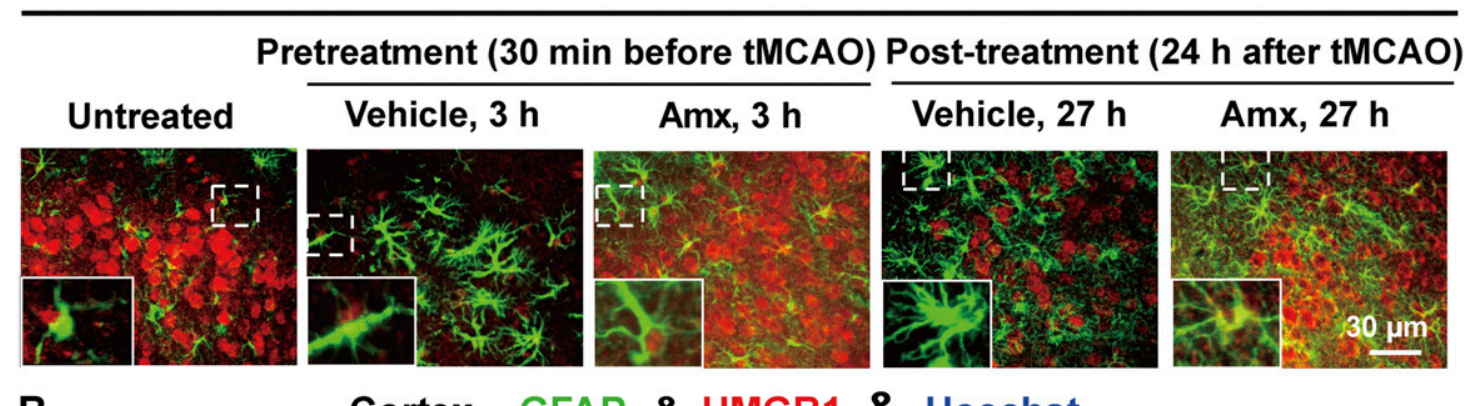

B

Cortex, GFAP \& HMGB1 \& Hoechst

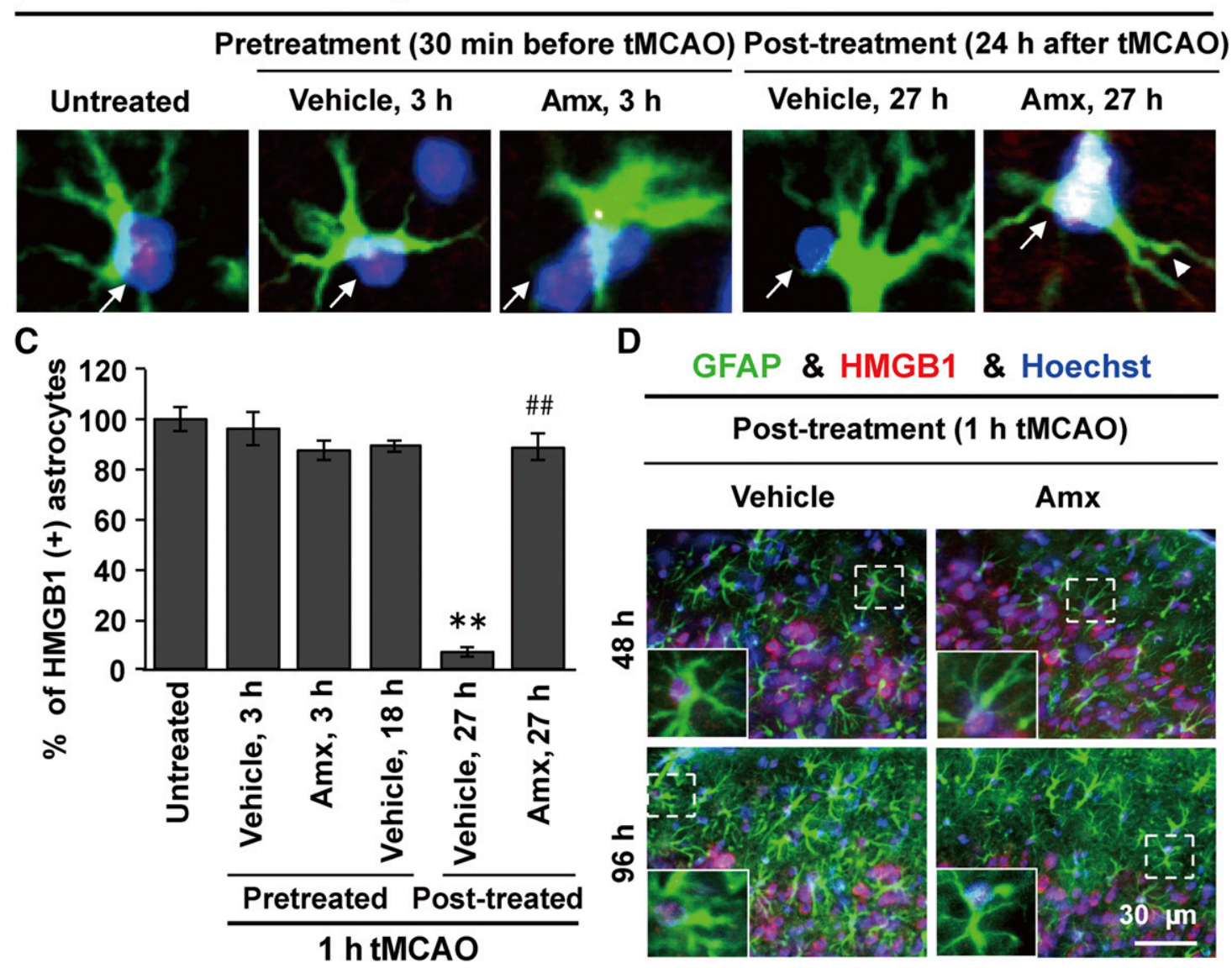

Fig. 4. Amlexanox blocks ischemia-induced release of HMGB1 from astrocytes. (A) Mice were pretreated with amlexanox (Amx) at a dose of $10 \mu \mathrm{g} / 5 \mu \mathrm{l}$ (i.c.v.) 30 minutes before 1-hour tMCAO, and immunostaining of GFAP and HMGB1 (GFAP, green; HMGB1, red) in the somatosensory cortex using coronal brain sections was performed at 3 hours after tMCAO. Following Amx post-treatment ( $10 \mu \mathrm{g} / 5 \mu \mathrm{l}$, i.c.v.) at 24 hours after 1-hour tMCAO, immunostaining of GFAP and HMGB1 was performed at 27 hours after tMCAO. Insets indicate the higher magnification view of HMGB1 signals in astrocytes noted by dotted squares in (A). (B) Triple immunostaining (GFAP, green; HMGB1, red; Hoechst, blue) showed the higher magnification view of HMGB1 localization in astrocytes at 0 (untreated), 3 hours after tMCAO (ischemic vehicle or Amx-pretreated mice), and 27 hours after tMCAO (vehicle or Amx post-treated mice). Nuclear and cytoplasmic HMGB1 signals in astrocytes are indicated by arrow and arrowhead, respectively. (C) Quantitative analysis of HMGB1-positive astrocytes in the cortex at 3,18 , and 27 hours after 1-hour tMCAO. Data are means \pm S.E.M. One-way ANOVA, Dunnett's multiple comparison tests, $* * P<0.01$ vs. untreated mice, ${ }^{\# \#} P<0.01$ vs. ischemic vehicle (27 hours); $n=3$ in each group. (D) Following Amx post-treatment at 24 hours after 1 hour tMCAO, HMGB1 signals were observed in the nuclei of cortical astrocytes at 48 or 96 hours after 1 hour tMCAO in both ischemic vehicle and Amx-treated mice. Insets indicate the higher magnification view of HMGB1 signals in astrocytes noted by dotted squares in (D); $n=3$ in each group.

tMCAO when mice were post-treated with $3 \mu \mathrm{g}$ anti-HMGB1 antibody (Fig. 8E).

\section{Discussion}

The present study includes the following major findings: 1) Pre-treatment with amlexanox 30 minutes before tMCAOtype cerebral ischemia reversibly blocks HMGB1 release from neurons at 3 hours after the ischemic stress, 2) post-treatment with amlexanox 24 hours after cerebral ischemia reversibly blocks HMGB1 release only from astrocytes at 27 hours after ischemia, 3) post-treatment with amlexanox protects the brain from ischemic damage, but as a pretreatment it fails to show any protection, and 4) post-treatment with an anti-HMGB1 antibody 24 hours after cerebral ischemia ameliorates ischemic brain damage. 
$\underline{A}$

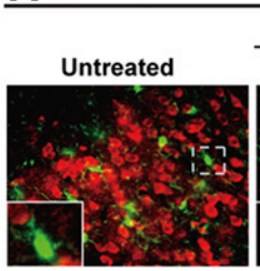

B

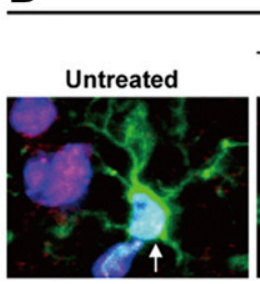

C
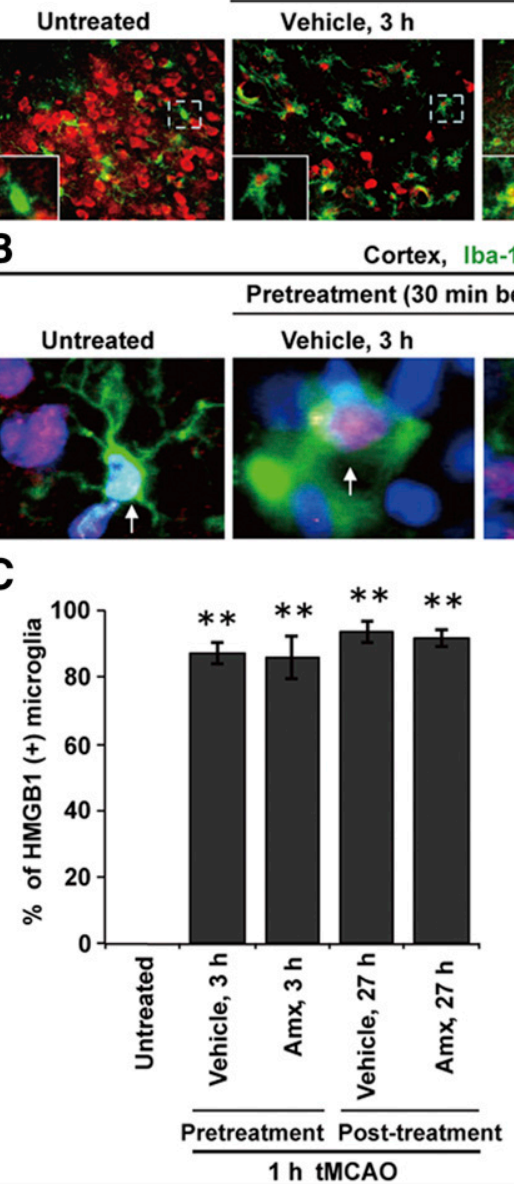

Cortex, Iba-1 \& HMGB1

Pretreatment ( $30 \mathrm{~min}$ before $1 \mathrm{~h} \mathrm{tMCAO}$ )

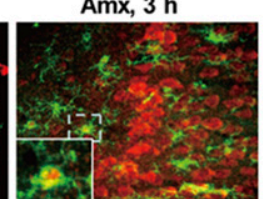

Cortex, Iba-1 \& HMGB1 \& Hoechst

Pretreatment ( $30 \mathrm{~min}$ before $1 \mathrm{~h}$ tMCAO)

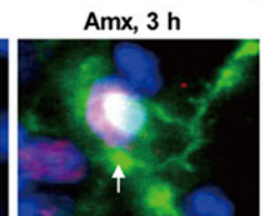

D

Cortex, Iba-1 \& HMGB1 \& Hoechst
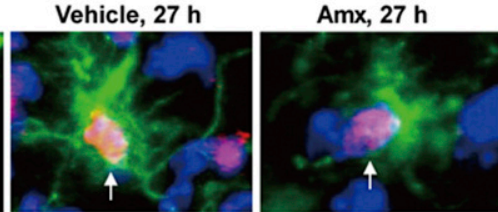

Post-treatment (1 h tMCAO)
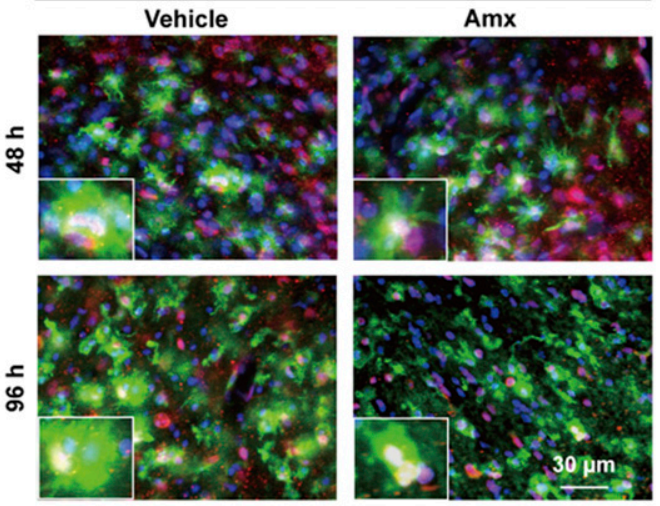

Fig. 5. Cerebral ischemia-induced expression of HMGB1 in microglia. (A) Mice were pretreated with amlexanox (Amx) at a dose of $10 \mu \mathrm{g} / 5 \mu \mathrm{l}$ (i.c.v.) 30 minutes before 1 hour tMCAO and immunostaining of Iba-1 and HMGB1 (Iba-1, green; HMGB1, red) in the somatosensory cortex using coronal brain sections was performed at 3 hours after tMCAO. Following Amx post-treatment ( $10 \mu \mathrm{g} / 5 \mu$ l, i.c.v. $)$ at 24 hours after 1-hour tMCAO, immunostaining of Iba-1 and HMGB1 was performed at 27 hours after tMCAO. Insets indicate higher magnification view of immunoreactive-HMGB1 in Iba-1-positive microglia noted by dotted squares. (B) Triple immunostaining (Iba-1, green; HMGB1, red; Hoechst, blue) show the higher magnification view of HMGB1 localization in microglia at 0 (untreated) and 3 hours after tMCAO (vehicle- or Amx-pretreated mice), and 27 hours after tMCAO (ischemic vehicle or Amx post-treated mice). (C) Quantitative analysis of HMGB1-positive microglia in the somatosensory cortex. Data are means \pm S.E.M. One-way ANOVA, Dunnett's multiple comparison tests, $* * P<0.01$ vs. untreated mice. (D) Following Amx treatment $(10 \mu \mathrm{g} / 5 \mu \mathrm{l}$, i.c.v. $)$ at 24 hours after 1-hour tMCAO, immunoreactive HMGB1 was still observed in the nuclei of Iba-1-positive microglia in the cortex at 48 or 96 hours after 1-hour tMCAO. Insets in (D) indicate higher magnification view of HMGB1 expression in microglia noted by dotted squares; $n=3$ in each group.

HMGB1 is released from neurons, some astrocytes, and microglia in response to ischemic stress (Hayakawa et al., 2008; Qiu et al., 2008), but it remains to be determined how the HMGB1 release occurs following cerebral ischemia in terms of time and cell-type specificity, and whether the release has pathophysiological roles in the post-ischemic brain. In the present study, immunohistochemistry showed that HMGB1 was completely released from nuclei of neurons at 3 hours after cerebral ischemia (1-hour tMCAO). On the other hand, HMGB1 was not released from astrocytes in the early phase of cerebral ischemia. It was found that neurons are the principal sources of HMGB1 in the early phase of brain ischemia/injury and this neuron-derived HMGB1 functions as an early upstream cytotoxic factor to cause ischemia-induced brain damage (Qiu et al., 2008;
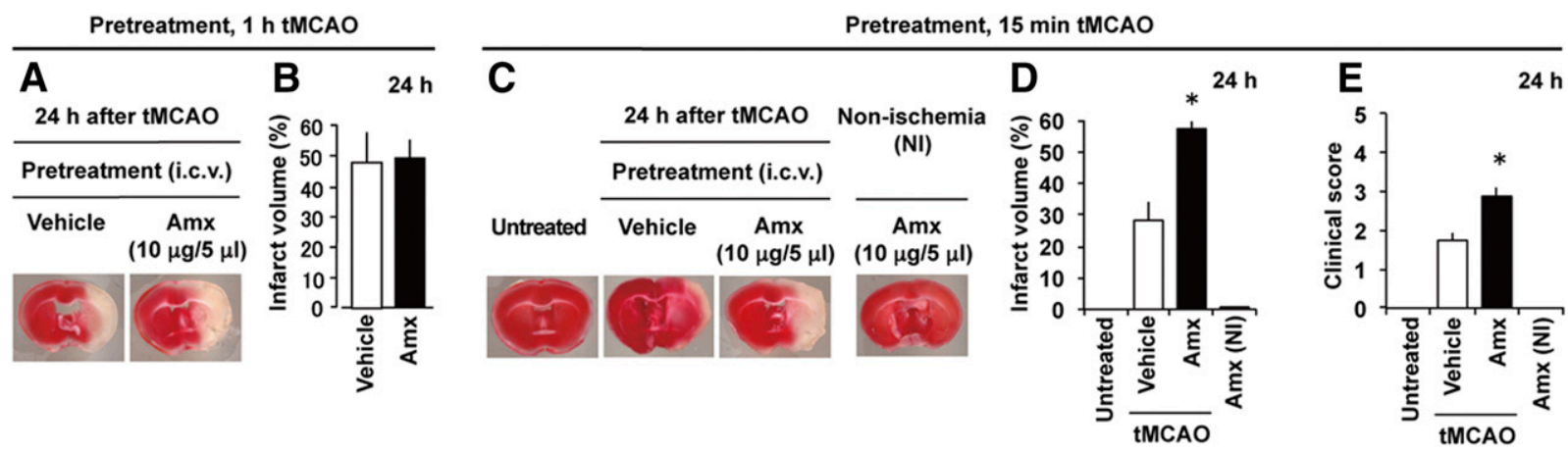

Fig. 6. Amlexanox pretreatment exacerbates the ischemia-induced damage. (A and B) Following amlexanox (Amx) pretreatment (10 $\mu \mathrm{g} / 5 \mu \mathrm{l}$, i.c.v.) 30 minutes before 1-hour tMCAO, TTC staining (A) and infarct volume measurement (B) were performed at 24 hours after tMCAO. Data are means \pm S.E.M. Student's $t$ test (not significant); $n=3$ in each group. (C-E) Mice were pretreated with Amx (10 $\mu \mathrm{g} / 5 \mu \mathrm{l}$, i.c.v.) 30 minutes before 15 -minute tMCAO. Nonischemic (NI) mice also received Amx (10 $\mu \mathrm{g} / 5 \mu \mathrm{l}$, i.c.v.). TTC staining (C), infarct volume measurement (D), and clinical score (E) were performed at 24 hours after tMCAO. Data are means \pm S.E.M. $* P<0.05$ vs. ischemic vehicle. Untreated, $n=3$; ischemic vehicle, $n=6$; Amx + tMCAO, $n$ $=6 ;$ Amx + nonischemic $(\mathrm{NI}), n=3$. 
A

$48 \mathrm{~h}$ after tMCAO

Injection (i.c.v.) $24 \mathrm{~h}$ after $\mathbf{3 0}$ min tMCAO
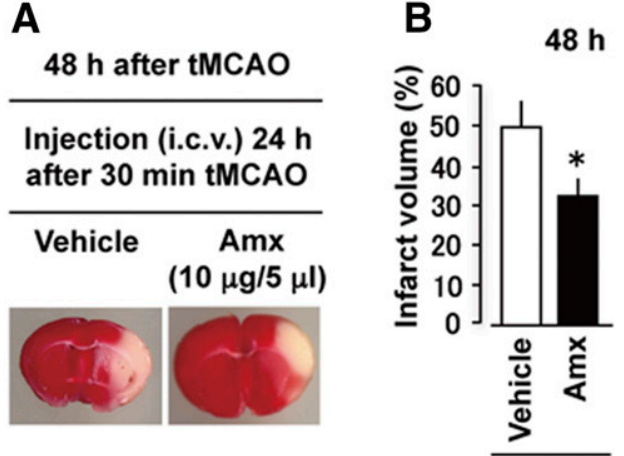

Injection (i.c.v.)

$24 \mathrm{~h}$ after $30 \mathrm{~min} \mathrm{tMCAO}$
E

$72 \mathrm{~h}$ after $\mathrm{tMCAO}$

Injection (i.c.v.) $48 \mathrm{~h}$
after 30 min tMCAO

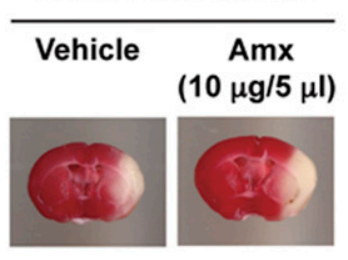

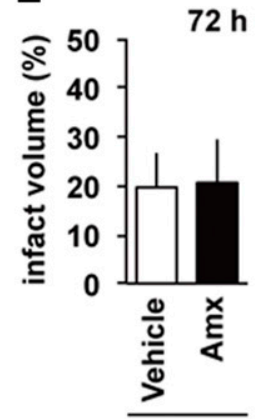

Injection (i.c.v.)
$48 \mathrm{~h}$ after $30 \mathrm{~min} \mathrm{tMCAO}$
C

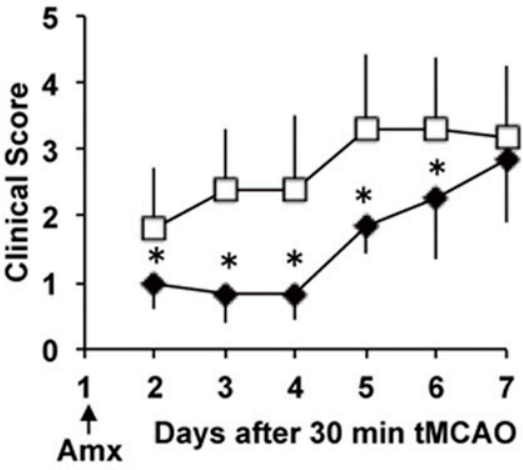

F

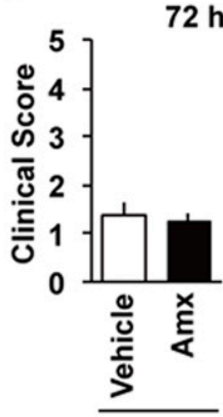

Injection (i.c.v.)

$48 \mathrm{~h}$ after $\mathbf{3 0} \mathrm{min}$ tMCAO
Fig. 7. Late treatment with amlexanox protects the brain from ischemic damage. (A and B) Following amlexanox (Amx) posttreatment $(10 \mu \mathrm{g} / 5 \mu \mathrm{l}$, i.c.v. $)$ at 24 hours after 30-minute tMCAO, TTC staining (A) and infarct volume measurement (B) were performed at 48 hours after ischemia. Data are means \pm S.E.M. Student's $t$ test, ${ }^{*} P<$ 0.05 vs. ischemic vehicle; $n=3$ in each group. (C) Following Amx post-treatment at 24 hours after 30 -minute tMCAO, evaluation of clinical score was performed every day for 7 days after tMCAO. Data are means \pm S.E.M. $* P<0.05$ vs. ischemic vehicle; $n=$ 6 in each group. (D-F) Following Amx posttreatment $(10 \mu \mathrm{g} / 5 \mu$ l, i.c.v. $)$ at 48 hours after 30-minute tMCAO, TTC staining (D), infarct volume measurement (E), and clinical score evaluation (F) were performed at 72 hours after tMCAO. Data are means \pm S.E.M. Student's $t$ test, $* P<0.05$ vs. ischemic vehicle; $* P<0.05$ vs. ischemic vehicle; $n=4$ in each group.
Sun et al., 2014). A previous study reported that HMGB1 is absent in microglia in the normal mouse brain, whereas brain microglia express HMGB1 following seizure (Maroso et al., 2010). Consistent with this, we observed the absence of immunoreactive
HMGB1 in microglia in the normal mouse brain. By contrast, HMGB1 expression appeared in microglia as early as 3 hours after cerebral ischemia, but no HMGB1 was released from microglia in this early phase of cerebral ischemia. These findings
A

$48 \mathrm{~h}$ after $30 \mathrm{~min}$ tMCAO

\begin{tabular}{ccc}
\hline \multicolumn{3}{c}{ Injection (i.c.v.) $24 \mathrm{~h}$ after tMCAO } \\
\hline Vehicle & $\begin{array}{c}\alpha-\mathrm{HMGB} 1 \\
(1 \mu \mathrm{g} / 5 \mu \mathrm{l})\end{array}$ & $\begin{array}{c}\alpha-\mathrm{HMGB} 1 \\
(3 \mu \mathrm{g} / 5 \mu \mathrm{l})\end{array}$ \\
\hline & & \\
\hline & &
\end{tabular}

C

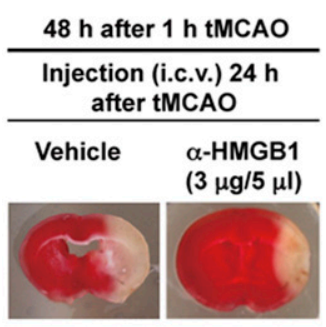

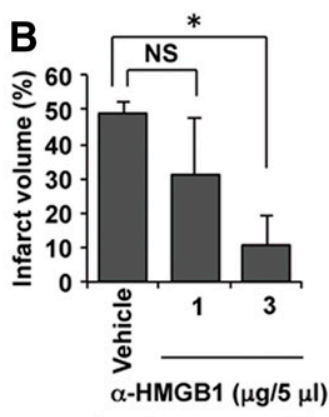

$48 \mathrm{~h}$ after $30 \mathrm{~min} \mathrm{tMCAO}$

E

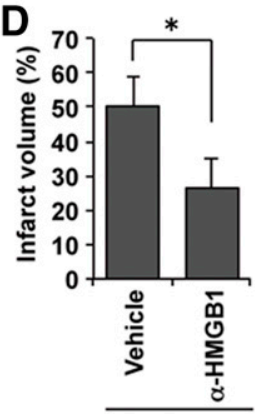

$48 \mathrm{~h} \overline{\text { after } 1 \mathrm{~h} \text { tMCAO }}$

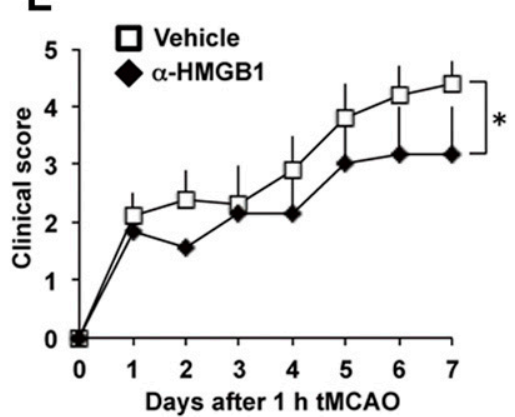

Fig. 8. Late treatment with anti-HMGB1 antibody inhibits ischemia-induced brain damage. (A and B) Following treatment with antiHMGB1 antibody ( $\alpha$-HMGB1) at a dose of 1 or $3 \mu \mathrm{g} / 5 \mu \mathrm{l}$ (i.c.v.) at 24 hours after 30 -minute tMCAO, TTC staining (A) and infarct volume measurement (B) were performed at 48 hours after tMCAO. Data are means \pm S.E.M. $* P<0.05$ vs. ischemic vehicle; $n=3$ in each group. (C and D) Following $\alpha$-HMGB1 treatment (3 $\mu \mathrm{g} / 5 \mu$ l, i.c.v.) at 24 hours after lethal cerebral ischemia (1-hour tMCAO), TTC staining (C) and infarct volume measurement (D) were performed at 48 hours after 1-hour tMCAO. Data are means \pm S.E.M. Student's $t$ test, $* P<0.05$ vs. ischemic vehicle. $n=3$ in each group. (E) Following $\alpha$-HMGB1 treatment $(3 \mu \mathrm{g} / 5 \mu$ l, i.c.v. $)$ at 24 hours after 1-hour tMCAO, clinical score was assayed every day for 7 days after tMCAO. Data are means \pm S.E.M. $* P<0.05$ vs. ischemic vehicle; ischemic vehicle, $n=5 ; \alpha$-HMGB1 + tMCAO, $n=6$. 
suggest that neurons are more sensitive to ischemia than astrocytes or microglia in terms of HMGB1 release in the early phase of cerebral ischemia. HMGB1, on the other hand, was released from astrocytes but not neurons at 27 hours after tMCAO. It is still unclear why astrocytes are more sensitive to ischemia than neurons in terms of HMGB1 release in the lateischemic phase.

On the other hand, cerebral ischemia-induced HMGB1 release from astrocytes was reversibly blocked by late treatment (24 hours after tMCAO) with amlexanox, without any effect on immunoreactive HMGB1 in neurons and microglia. On the basis of these results, we attempted to evaluate the effect of amlexanox post-treatment against ischemia-induced brain damage. Our findings suggested that late treatment with amlexanox at 24 hours, but not at 48 hours after ischemia (30-minute tMCAO) significantly inhibited ischemia-induced brain damage in terms of TTC, motor dysfunction, and lethality. Amlexanox-induced (late treatment) protection of ischemic brain was confirmed by the late treatment (i.c.v.) with neutralizing anti-HMGB1 antibody at 24 hours after 30-minute or 1 hour tMCAO, in which ischemic brain damage was significantly ameliorated by the late treatment with antiHMGB1 antibody. Moreover, our finding with the antiHMGB1 antibody experiment is consistent with previous studies in brain ischemia (Kim et al., 2006; Liu et al., 2007; Muhammad et al., 2008). Thus, late release of HMGB1 particularly from astrocytes contributes to ischemic brain damage. Pretreatment with amlexanox 30 minutes before tMCAO reversibly blocked cerebral ischemia-induced HMGB1 release from brain neurons at 3 hours after tMCAO, without any effect on immunoreactive HMGB1 in nuclei of astrocytes and microglia. On the basis of these findings, we observed that ischemia-induced brain damage was significantly worse at 24 hours after the ischemic stress in amlexanox-pretreated mice, as shown by TTC staining and behavioral experiments.

Recently, we demonstrated that $\operatorname{ProT} \alpha$, a signal peptideless nuclear protein, was nonclassically released from the nuclei of cultured C6 astroglioma cells through interaction with cargo molecule S100A13 following ischemia, and this extracellular release of ProT $\alpha$ was reversibly blocked by amlexanox (Matsunaga and Ueda, 2010; Ueda et al., 2012). Most recently, we also reported that ProT $\alpha$ was nonclassically released from the nuclei of neurons as early as 3 hours after cerebral ischemia (1-hour tMCAO) and this ProT $\alpha$ release is reversibly blocked by the pretreatment with amlexanox (Halder et al., 2012). On the other hand, microglia did not release ProT $\alpha$ in the presence of cerebral ischemia, owing to absence of S100A13 expression in this glial cell (Halder et al., 2012). Taken together, it is interesting to speculate that the initiation of ischemia-induced mechanism for HMGB1 release from neurons is less important than that of the release of neuroprotective molecules such as ProT $\alpha$ in the early phase of cerebral ischemia. Furthermore, HMGB1, which was released from astrocytes in the late-ischemic phase, may cause the deterioration of ischemic brain damage by induction of cytotoxic cascades. Since $\operatorname{ProT} \alpha$, a neuroprotective DAMPs, is expressed in neurons and astrocytes on day 1 ( 27 hours) after cerebral ischemia independently of amlexanox (Halder et al., 2012), the blockade of HMGB1 release from astrocytes either by amlexanox or anti-HMGB1 antibody late treatment may be a novel approach to protection against ischemic brain damage.
Further studies would be necessary to examine the effects of amlexanox on HMBG1 level in the CSF under cerebral ischemia, the effects of amlexanox late treatment on brain HMGB1 levels at later time points, and whether the gene silencing of amlexanox targets such as S100A13 mimics the amlexanox effects. In addition, it would also be interesting to see how pretreatment with anti-HMGB1 antibody affects ischemia-induced brain damage, since the neuroprotective DAMP molecule ProT $\alpha$ is also released upon ischemic stress in an amlexanox-reversible manner (Halder et al., 2012). As many other DAMPs molecules would also be released upon acute ischemic stress, detailed analysis of the contribution of released HMGB1 needs more extensive analysis, including the effects of gene silencing of HMGB1.

Amlexanox reversibly blocked ischemia-induced early release of HMGB1 from neurons and late release from astrocytes, but we do not know how HMGB1 release is inhibited by amlexanox. As there are reports that amlexanox has multiple pharmacological actions (Bell, 2005; Zhang et al., 2015), the molecular-based mechanisms underlying ischemia-induced nonclassical release of HMGB1 may not be simple. However, as amlexanox inhibits the nonclassical release of various DAMPs molecules, including IL- $1 \alpha$, fibroblast growth factor (FGF)-1 and ProT $\alpha$ (Prudovsky et al., 2003, 2008; Matsunaga and Ueda, 2008, 2010), which commonly use S100A13 as a cargo protein, the study of interaction between HMGB1 and S100A13 represents an obvious approach to clarify the mechanisms underlying HMGB1 release.

In conclusion, pretreatment with amlexanox blocked cerebral ischemia-induced early release of HMGB1 from neurons but failed to protect the brain from ischemic damage. Late treatment with amlexanox blocked HMGB1 release from astrocytes 27 hours after ischemia and subsequently limited the extent of ischemic brain damage. In addition, ischemiainduced brain damage was ameliorated by late treatment with the anti-HMGB1 antibody. Although amlexanox is clinically used as a safe antiallergy medicine, this compound has multiple cellular targets in the periphery of the body. Therefore, the present discovery of pharmacological regulation of delayed and harmful HMGB1 release may provide a new strategy for developing antistroke medicines that possess a wide therapeutic time frame.

\section{Acknowledgments}

The authors thank Dr. H. Matsunaga, Dr. Y. Matsushita, H. Yamaguchi, and J. Sugimoto for technical help and acknowledge Takeda Pharmaceutical Company Ltd. for providing amlexanox. The authors also thank Dr. R. Milner (Associate Professor, The Scripps Research Institute, La Jolla) for checking the English grammar throughout the manuscript.

\section{Authorship Contributions \\ Participated in research design: Ueda, Halder. Conducted experiments: Halder. \\ Performed data analysis: Halder. \\ Contributed new reagents or analytic tools: Ueda. \\ Wrote or contributed to the writing of the manuscript: Ueda, Halder.}

\section{References}

Andersson U and Tracey KJ (2011) HMGB1 is a therapeutic target for sterile inflammation and infection. Annu Rev Immunol 29:139-162.

Bell J (2005) Amlexanox for the treatment of recurrent aphthous ulcers. Clin Drug Investig 25:555-566. 
Egashira N, Hayakawa K, Mishima K, Kimura H, Iwasaki K, and Fujiwara M (2004) Neuroprotective effect of gamma-glutamylethylamide (theanine) on cerebral infarction in mice. Neurosci Lett 363:58-61.

Erlandsson Harris H and Andersson U (2004) Mini-review: the nuclear protein HMGB1 as a proinflammatory mediator. Eur J Immunol 34:1503-1512.

Fujita R and Ueda H (2007) Prothymosin-alpha1 prevents necrosis and apoptosis following stroke. Cell Death Differ 14:1839-1842.

Fujita R, Ueda M, Fujiwara K, and Ueda H (2009) Prothymosin-alpha plays a defensive role in retinal ischemia through necrosis and apoptosis inhibition. Cell Death Differ 16:349-358.

Halder SK, Matsunaga H, Ishii KJ, and Ueda H (2015) Prothymosin-alpha preconditioning activates TLR4-TRIF signaling to induce protection of ischemic retina. J Neurochem 135:1161-1177.

Halder SK, Matsunaga H, and Ueda H (2012) Neuron-specific non-classical release of prothymosin alpha: a novel neuroprotective damage-associated molecular patterns. J Neurochem 123:262-275.

Halder SK, Yano R, Chun J, and Ueda H (2013) Involvement of LPA1 receptor signaling in cerebral ischemia-induced neuropathic pain. Neuroscience 235:10-15

Harris HE, Andersson U, and Pisetsky DS (2012) HMGB1: a multifunctional alarmin driving autoimmune and inflammatory disease. Nat Rev Rheumatol 8:195-202.

Hayakawa K, Mishima K, Nozako M, Hazekawa M, Mishima S, Fujioka M, Orito K Egashira N, Iwasaki K, and Fujiwara M (2008) Delayed treatment with minocycline ameliorates neurologic impairment through activated microglia expressing a high-mobility group box1-inhibiting mechanism. Stroke 39:951-958.

Hjelm F, Tran B, and Fredriksson S (2011) Sensitive detection of cytokines in 1- $\mu$ l serum samples using Proseek®. Nat Methods 8:iii; DOI: 10.1038/nmeth.f.348.

Kim JB, Sig Choi J, Yu YM, Nam K, Piao CS, Kim SW, Lee MH, Han PL, Park JS, and Lee JK (2006) HMGB1, a novel cytokine-like mediator linking acute neuronal death and delayed neuroinflammation in the postischemic brain. $J$ Neurosci 26 $6413-6421$.

Klune JR, Dhupar R, Cardinal J, Billiar TR, and Tsung A (2008) HMGB1: endogenous danger signaling. Mol Med 14:476-484.

Landriscina M, Prudovsky I, Mouta Carreira C, Soldi R, Tarantini F, and Maciag T (2000) Amlexanox reversibly inhibits cell migration and proliferation and induces the Srcdependent disassembly of actin stress fibers in vitro. J Biol Chem 275:32753-32762.

Lange SS, Mitchell DL, and Vasquez KM (2008) High mobility group protein B1 enhances DNA repair and chromatin modification after DNA damage. Proc Natl Acad Sci USA 105:10320-10325.

Liu K, Mori S, Takahashi HK, Tomono Y, Wake H, Kanke T, Sato Y, Hiraga N, Adachi N, Yoshino T, et al. (2007) Anti-high mobility group box 1 monoclonal antibody ameliorates brain infarction induced by transient ischemia in rats. FASEB $J$ 21:3904-3916.

Liu L and Duff K (2008) A technique for serial collection of cerebrospinal fluid from the cisterna magna in mouse. $J$ Vis Exp 21:960.

Lotze MT and Tracey KJ (2005) High-mobility group box 1 protein (HMGB1): nuclear weapon in the immune arsenal. Nat Rev Immunol 5:331-342.

Maeda S, Sasaki K, Halder SK, Fujita W, and Ueda H (2016) Neuroprotective DAMPs member prothymosin alpha has additional beneficial actions against cerebral ischemia-induced vascular damages. J Pharmacol Sci 132:100-104.

Maroso M, Balosso S, Ravizza T, Liu J, Aronica E, Iyer AM, Rossetti C, Molteni M, Casalgrandi M, Manfredi AA, et al. (2010) Toll-like receptor 4 and high-mobility group box-1 are involved in ictogenesis and can be targeted to reduce seizures. Nat Med 16:413-419.

Matsunaga $\mathrm{H}$ and Ueda $\mathrm{H}$ (2008) Synergistic $\mathrm{Ca} 2+$ and $\mathrm{Cu} 2+$ requirements of the FGF1-S100A13 interaction measured by quartz crystal microbalance: an initial step in amlexanox-reversible non-classical release of FGF1. Neurochem Int 52:1076-1085.
Matsunaga $\mathrm{H}$ and Ueda $\mathrm{H}$ (2010) Stress-induced non-vesicular release of prothymosin- $\alpha$ initiated by an interaction with $\mathrm{S} 100 \mathrm{~A} 13$, and its blockade by caspase- 3 cleavage. Cell Death Differ 17:1760-1772.

Mohan SK and Yu C (2011) The IL1alpha-S100A13 heterotetrameric complex structure: a component in the non-classical pathway for interleukin 1alpha secretion. J Biol Chem 286:14608-14617.

Mouta Carreira C, LaVallee TM, Tarantini F, Jackson A, Lathrop JT, Hampton B Burgess WH, and Maciag T (1998) S100A13 is involved in the regulation of fibroblast growth factor- 1 and $\mathrm{p} 40$ synaptotagmin-1 release in vitro. $J$ Biol Chem 273:22224-22231.

Muhammad S, Barakat W, Stoyanov S, Murikinati S, Yang H, Tracey KJ, Bendszus M, Rossetti G, Nawroth PP, Bierhaus A, et al. (2008) The HMGB1 receptor RAGE mediates ischemic brain damage. $J$ Neurosci 28:12023-12031.

Okada M, Itoh H, Hatakeyama T, Tokumitsu H, and Kobayashi R (2003) Hsp90 is a direct target of the anti-allergic drugs disodium cromoglycate and amlexanox. Biochem J 374:433-441.

Palumbo R, De Marchis F, Pusterla T, Conti A, Alessio M, and Bianchi ME (2009) Src family kinases are necessary for cell migration induced by extracellular HMGB1. $J$ Leukoc Biol 86:617-623.

Prudovsky I, Mandinova A, Soldi R, Bagala C, Graziani I, Landriscina M, Tarantini F, Duarte M, Bellum S, Doherty H, et al. (2003) The non-classical export routes: FGF1 and IL-1alpha point the way. J Cell Sci 116:4871-4881.

Prudovsky I, Tarantini F, Landriscina M, Neivandt D, Soldi R, Kirov A, Small D, Kathir KM, Rajalingam D, and Kumar TK (2008) Secretion without Golgi. J Cell Biochem 103:1327-1343.

Qiu J, Nishimura M, Wang Y, Sims JR, Qiu S, Savitz SI, Salomone S, and Moskowitz MA (2008) Early release of HMGB-1 from neurons after the onset of brain ischemia. J Cereb Blood Flow Metab 28:927-938.

Stros M (2010) HMGB proteins: interactions with DNA and chromatin. Biochim Biophys Acta 1799:101-113.

Sun Q, Wu W, Hu YC, Li H, Zhang D, Li S, Li W, Li WD, Ma B, Zhu JH, et al. (2014) Early release of high-mobility group box 1 (HMGB1) from neurons in experimenta subarachnoid hemorrhage in vivo and in vitro. $J$ Neuroinflammation 11:106.

Tang D, Kang R, Zeh HJ, III, and Lotze MT (2011) High-mobility group box 1, oxidative stress, and disease. Antioxid Redox Signal 14:1315-1335.

Ueda H, Matsunaga H, and Halder SK (2012) Prothymosin $\alpha$ plays multifunctiona cell robustness roles in genomic, epigenetic, and nongenomic mechanisms. Ann NY Acad Sci 1269:34-43.

Wang J, Dotson AL, Murphy SJ, Offner H, and Saugstad JA (2015) Adoptive transfer of immune subsets prior to MCAO does not exacerbate stroke outcome in splenectomized mice. J Syst Integr Neurosci 1:20-28.

Yang QW, Wang JZ, Li JC, Zhou Y, Zhong Q, Lu FL, and Xiang J (2010) Highmobility group protein box-1 and its relevance to cerebral ischemia. $J$ Cereb Blood Flow Metab 30:243-254.

Yu M, Wang H, Ding A, Golenbock DT, Latz E, Czura CJ, Fenton MJ, Tracey KJ, and Yang H (2006) HMGB1 signals through toll-like receptor (TLR) 4 and TLR2. Shock 26:174-179.

Zhang Y, Guan H, Li J, Fang Z, Chen W, and Li F (2015) Amlexanox suppresses osteoclastogenesis and prevents ovariectomy-induced bone loss. Sci Rep 5:13575.

Address correspondence to: Dr. Hiroshi Ueda, Department of Pharmacology and Therapeutic Innovation, Nagasaki University Graduate School of Biomedical Sciences, 1-14 Bunkyo-machi, Nagasaki 852-8521, Japan. E-mail: ueda@nagasaki-u.ac.jp 